\title{
Respiration
}

\begin{tabular}{l}
\hline 12.10.-15.10.2009 \\
Tehran \\
Iran \\
\hline 19.10.-23.10.2009 \\
Ancona \\
Italy \\
\end{tabular}

31.10.-5.11.2009

San Diego, Calif. USA

12.11.-14.11.2009

Amsterdam

The Netherlands

\subsection{1.-18.11.2009}

Seoul

South Korea

16.11.-20.11.2009
Ancona
Italy

17.11.-19.11.2009

Tehran

Iran

\subsection{1.-27.11.2009 \\ Marseille}

France

\subsection{1.-27.11.2009 \\ Barcelona \\ Spain}

17.3.-20.3.2010

Hannover

Germany

29.4.-30.4.2010

Lausanne

Switzerland
The 4th International Congress on Pulmonary Disease, Intensive Care and Tuberculosis

Weeks in Interventional Pulmonolgy

Flexible and Rigid Bronchoscopy

\section{CHEST 2009}

Future CHEST Meetings:

CHEST 2010 - 30.10.-4.11.2010, Vancouver, B.C., Canada

CHEST 2011 - 22.10.-27.10.2011, Honolulu, Hawaii, USA

ERS School Course on Medical Aerosols, jointly organised with ISAM

14th Congress of the APSR and 3rd Joint Congress of the APSR/ACCP

Weeks in Interventional Pulmonolgy

Flexible and Rigid Bronchoscopy

3rd Iranian Asthma Meeting - 7th Biennial Seminar of the Iranian Society of Asthma and Allergy (ISAA)

\section{ERS School Course on Thoracoscopy and Pleural Techniques}

International Joint Meeting on Thoracic Surgery: 6th International Meeting on General Thoracic Surgery, 5th International Workshop on Surgical Exploration of the Mediastinum and Systematic Nodal Dissection, and 3rd Scientific Meeting of the Bronchogenic Carcinoma Cooperative Group of the Spanish Society of Pneumology and Thoracic Surgery

\section{Kongress der Deutschen Gesellschaft für Pneumologie und} Beatmungsmedizin

Jahresversammlung der Schweizerischen Gesellschaft für Pneumologie
Information: National Research Institute of Tuberculosis and Lung Disease, Tehran

E-Mail fic.nritld@gmail.com, http://congress.nritld.ac.ir

Information: AIPO Ricerche

Via Antonio da Recanate, 2

IT-20124 Milano (Italy)

Tel. +39023659 0354, Fax +390236590360

E-Mail s.gasparini@fastnet.it www.eabip.org

Information: ACCP Member Service

3300 Dundee Road, Northbrook, IL 60062 (USA)

Tel. +1 847498 1400, Fax +1 8474985460

E-Mail registrationschestnet.org, www.chestnet.org

Information: ERS Headquarters, 4, Avenue Ste. Luce $\mathrm{CH}-1003$ Lausanne (Switzerland)

Tel. +4121213 0101, Fax +41212130100

E-Mail info@ersnet.org,www.ersnet.org

Information: E-Mail apsr2009@intercom.co.kr www.apsr2009.org

Information: AIPO Ricerch

Via Antonio da Recanate, 2

IT-20124 Milano (Italy)

Tel. +39023659 0354, Fax +390236590360

E-Mail s.gasparini@fastnet.it

www.eabip.org

Information: Immunology, Asthma and Allergy Research Institute (IAARI), Children Medical Center 62 Dr. Gharib St., Keshavarz Blvd, P.O. Box 14185-863 Tehran 14194 (Iran)

Tel. +98 216693 8545, Fax +982166428995

E-Mail isaacong@tums.ac.ir, http://isaacong.tums.ac.ir

Information: ERS Headquarters, 4, Avenue Ste. Luce CH-1003 Lausanne (Switzerland)

Tel. +41 21213 0101, Fax +41212130100

E-Mail info@ersnet.org, www.ersnet.org

Information: Acto Serveis, Barcelona

E-Mail oriol@actoserveis.com

www.thoracicsurgery2009.org

Information: www.dgp-kongress.de

Information: www.pneumo.ch 
14.5.-19.5.2010

New Orleans, La.

USA

5.6.-9.6.2010

London

UK

7.10.-9.10.2010

Graz

Austria

15.6.-18.6.2011

Maastricht

The Netherlands
ATS 2010 - American Thoracic Society

Future ATS Meeting:

13.5.-18.5.2011, Denver, Colo., USA

XXIX Congress of the European Academy of Allergology and Clinical Immunology

Jahrestagung 2010 der Österreichischen Gesellschaft für

Pneumologie (ÖGP)

WASOGBAL 2011
Information: www.thoracic.org

Information: Congrex Sweden AB, Stockholm

E-Mail eaaci2010@congrex.com

www.congrex.com

Information: Ärztezentrale Med. Info

Helferstorferstraße 4, AT-1014 Wien

Tel. +431531 1638, Fax +4315311661

E-Mail azmedinfo@media.co.at

www.ogp.at

Information: WASOGBAL 2011 Foundation

Maastricht Unversity Medical Centre

E-Mail ild.care@mumc.nl

www.wasogbal2011.nl 